\title{
British and Spanish Youths: How different are their eating habits?
}

\author{
M.P. Vílchez ${ }^{1}$ and L. Santos-Merx ${ }^{2}$ \\ ${ }^{1}$ Department of Education, Faculty of Social Sciences and Communication, Catholic University of Murcia, Spain and \\ ${ }^{2}$ Food and Human Nutrition, Newcastle University Singapore, Singapore
}

Growing up in a culture where the Mediterranean diet is prevalent such as in Spain, should be a protective factor against the influence of the obesogenic environment and help prevent poor food choices and habits, e.g. less consumption of sweetened beverages, fast food, confectionery and crisps ${ }^{(1)}$. The progressive globalisation of food products has contributed to a decreasing consumption of traditional healthy foods also in cultures other than the Mediterranean ${ }^{(2,3)}$ increasing the risk of developing obesity and its associated diseases. The aim of the present study is to contrast British and Spanish young to mid adolescents' adherence to healthy eating habits via a survey using the KIDMED Index ${ }^{(4)}$ and look for age and gender differences.

The sample included 1015 adolescents aged 12-14 years from Spanish $(n=604,50 \%$ males $)$ and British schools/youth clubs $(n=$ 411, $48 \%$ males). Surprisingly, adolescents from UK (Mean Rank $=539 \cdot 31)$ had a higher KIDMED Index $(Z=-2 \cdot 825 ; p=.005)$ than from Spain (Mean Rank $=486.69$ ) suggesting higher adherence to healthy eating. About half of participants from both countries were on the category Diet to improve (Figure 1). However, more British adolescents were placed in the category Good diet whilst more Spanish were in the category Poor $\left(\chi_{(2)}^{2}=15.758, p<.0005\right)$.

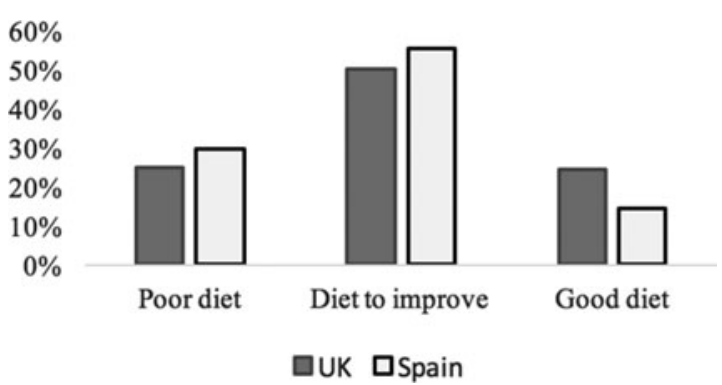

Fig. 1. British and Spanish adolescents' diet quality based on the KIDMED Index.

Indeed, significantly more Spanish reported having sweets, fast foods, pastries and skipping breakfast than British did. Nonetheless, more Spanish than British stated having fish, dairy, vegetables, nuts and olive oil whilst more British had pulses and starchy foods ( $p$ values $<.05$ ). Similarly, significant results were obtained when contrasting the same gender between both countries.

Majority of participants from both countries shared healthy habits such as consuming fruit and/or vegetables once to twice a day ( $p$ values $>.05$ ). Similar outcomes were obtained when contrasting the same gender between both countries.

Boys from Spain (Mean Rank $=320.38 .74 ; Z=-2.538 ; p=.011$ ) scored higher on the KIDMED Index than girls (Mean Rank= 284.62). This indicates these Spanish boys may adhere to healthy eating more than girls. For instance, pastries and fast foods were consumed more frequently by girls than boys $(p$ values $<.05)$. In the UK, and despite of both genders had nearly similar KIDMED Index score $(p>\cdot 05)$, more boys reported consuming pulses and fish whilst more girls skipped breakfast $(p$ values $<.05)$.

UK KIDMED Index and age shared a small negative but significant relationship $(\rho=-.150 ; p=.002)$. However, for Spain this relationship was significantly positive $(\rho=.084 ; p=.039)$. Despite being weak, these values suggest as age increases the diet quality for these British adolescents gets slightly worse whilst for the Spanish sample it improves a bit.

These results should be taken with caution, nonetheless justify further reinforcement of current health promotion strategies in place in UK and Spain aiming to improve diet quality of young people.

1. Trichopoulou A \& Lagiou P (1997) Nut Rev 55, 383-389.

2. Arriscado D, Muros JJ, Zabala M \& Dalmau JM (2014) Appetite 80, 28-34

3. Karlén J, Lowert Y, Chatziarsenis KM et al. (2008) Rural and Remote Health 8, 1034

4. Serra-Majem L, Ribas L, Ngo J et al. (2004) Public Health Nutr 7, 931-935. 